\title{
Efficient Simulation for Testing Loss of Separation Algorithms
}

\author{
Allan L. White \\ NASA Langley \\ Hampton, VA 23681 \\ 757-864-6199 \\ a.1.white@larc.nasa.gov
}

\begin{abstract}
Given increased air traffic and the possible use of autonomous vehicles, there is interest in the verification of separation algorithms. Because of the complexity of the environment and the presence of perturbations, a candidate approach is simulation and Monte Carlo. There are several desirable properties of the simulation: realism, portability, transparency, and efficiency. This paper is an initial effort using MATLAB to study a separation algorithm. An original separation algorithm was developed to make this project independent of any other effort. The algorithms and program were effective enough and efficient enough that the project will proceed. ${ }^{12}$
\end{abstract}

\section{TABLE OF CONTENTS}

1. INTRODUCTION ........................................... 1

2. DESCRIPTION OF FLIGHT PATHS ..................... 2

3. ANGLE OF APPROACH .................................... 2

4. DESCRIPTION OF ClosENESS DETECTION ................ 2

5. DESCRIPTION AND CONSTRUCTION OF A JOG ............. 3

6. TYPES OF COLLISION AVOIDANCE .......................4

7. DESCRIPTION OF SiMUlation SPACE ...................5

8. SiMUlation Runs ................................... 6

9. ElEMENTARY PRoBabILITY ANALYSIS ............... 7

10. Field Data and Conditional Probability .........7

11. FURTHER WORK ….................................... 8

12. SUMMARY ............................................ 8

REFERENCES ........................................... 8

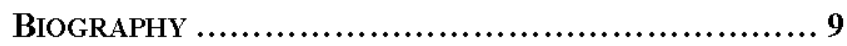

\section{INTRODUCTION}

This is an initial attempt at designing a separation algorithm for aircraft and checking its effectiveness by simulation. Two sub goals were to write the simulation in a popular program (MATLAB) for portability and to examine the efficiency of using a general purpose language for the simulation. Portability and using a common language were considered for the purpose of comparing various separation algorithms. It's desirable to write code that is easily examined since a feature of some large programs is that important details are buried in the code. Efficiency is needed because of the need to run many trials to establish

\footnotetext{
${ }^{1}$ U.S. Government work not protected by U.S. copyright

2 IEEEAC paper \# 1237, Version 2, Updated Oct 21, 2007
}

confidence in the separation algorithm.

The quest for simplicity, clarity, and efficiency does produce a dilemma: the material appears trivial. In addition, the algorithm and simulation are exercises in elementary mathematics: analytic geometry, trigonometry, and calculus. The goal for the future is to increase the realism while preserving the simplicity, clarity, and efficiency (the triviality).

As a first effort, there are a number of assumptions, not all of them completely justified. (1) Only two aircraft at a time are considered. (2) All aircraft have the same speed and maintain this speed. Aircraft do prefer to maintain a constant airspeed. (3) Everything is two-dimensional. (4) Since everything is two-dimensional, the only option for collision avoidance for closing aircraft on nearly the same flight path is the delay of one of the aircraft. It is assumed the delay can occur before the aircraft enters the flight space being considered. (5) The position and heading of all aircraft is precisely known. (6) Both aircraft know which one will make the collision-avoidance maneuver and what the maneuver will be. (7) Previous flight control efforts have gone well, and aircraft enter the airspace separated by at least the minimum distance. (8) All flight paths are sequences of straight-line segments.

Since the flight paths in this study are sequences of straightline segments, the heart of the program is a routine that decides if two particles moving on two different straight segments remain a minimum distance apart. This is described in section 4 .

When writing a program to simulate the random nearcollision of aircrafts, the question arises about which random distribution to use when generating flight paths. An authentic simulation must reproduce the distribution of flight paths in the field or show the form of the distribution does not matter. To the best of the author's knowledge, this remains an open problem.

Section two describes how flight paths, or segments of a flight path, are stored in the program. Section three discusses determining the angle of approach of the two aircraft. Section four covers detecting a loss of separation. Section five describes the avoidance maneuver. Section six covers deciding which avoidance maneuver to use. Section seven describes simulating near collision. Section eight gives the efficiency of the program. Section nine performs 
an elementary probability analysis. Section ten conducts a more refined probability analysis. Since this is an initial effort, some of the material below notes where improvements could be made.

\section{Description of Flight Path}

The flight path of any aircraft in this study is a sequence of directed straight line segments (vectors). A straight line segment is stored in the program as a $6 \times 1$ column vector that has the form.

\begin{tabular}{|l|}
\hline start time \\
\hline end time \\
\hline $\mathrm{x}$ position at start time \\
\hline $\mathrm{y}$ position at start time \\
\hline $\mathrm{x}$ position at end time \\
\hline $\mathrm{y}$ position at end time \\
\hline
\end{tabular}

A flight path consisting of more than one segment is stored as a multi-column vector with the segments in order from left to right.

If the program were re-written, the information vector would be enlarged to include the sine and cosine of the directed line segment. These are in constant use throughout the program, and the current program is constantly recomputing them

\section{ANGLe of APPRoACh}

The decision algorithm depends on the relative angle at which the two planes are approaching each other. A headon approach is where the angle of approach is between $\pi / 2$ and $3 \pi / 2$. This is determined by taking the cosine of the difference of the two flight path angles. If the cosine is negative the approach is head-on.

If the approach is head-on, the program needs to know if the second aircraft is approaching from the left or the right. Consider a difference angle - the angle of the aircraft that will perform the maneuver minus the angle of the other aircraft. If the sine of this difference angle is negative, the aircraft that will perform the maneuver is approaching from the left. If positive, from the right.

An approach angle of $\pi$ radians requires a different method which is illustrated in figure 1. In this figure, the aircraft that will maneuver is upper one.

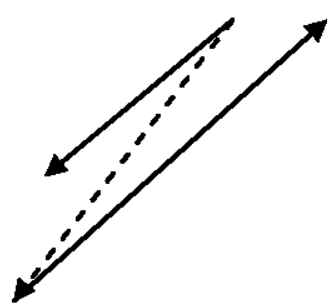

Figure 1 - Aircraft approaching at $\pi$ radians

For this case, take the vector from the start-point of the aircraft that maneuvers to the start point of the aircraft that will not maneuver. This is shown in the figure above by a dashed line. Subtract the angle of the vector of the maneuvering aircraft from the angle for the new vector. If the sine of the difference is positive, the maneuvering aircraft is to the left of the other aircraft. If negative, to the right. If zero, it is head-on.

\section{Description of Closeness Detection ROUTINES}

As will be seen below, it is necessary to examine two arbitrary flight path segments to determine if the aircrafts moving on them violate the separation requirement.

A complicating factor is that the start and end times for the two paths can be different. Hence, the actual comparison is made on the parts of the segments that begin with the larger starting time and end with the smaller end time. Once the relevant segments have been determined, the procedure becomes the minimum problem of calculus: find the zero value of the derivative and check the endpoints. For instance, given the vector description of two path segments for two different aircrafts:

\begin{tabular}{|l|}
\hline Start time $=1$ \\
\hline End time $=6$ \\
\hline$x$ position at time 1 \\
\hline y position at time 1 \\
\hline x position at time 6 \\
\hline y position at time 6 \\
\hline
\end{tabular}

\begin{tabular}{|l|}
\hline Start time $=3$ \\
\hline End time $=12$ \\
\hline$x$ position at time 3 \\
\hline$y$ position at time 3 \\
\hline$x$ position at time 12 \\
\hline$y$ position at time 12 \\
\hline
\end{tabular}


The program determines the flight segment for the two aircrafts from time 3 to time 6 . Each vector has the form:

\begin{tabular}{|l|}
\hline Start time $=3$ \\
\hline End time $=6$ \\
\hline$x$ position at time 3 \\
\hline$y$ position at time 3 \\
\hline$x$ position at time 6 \\
\hline$y$ position at time 6 \\
\hline
\end{tabular}

An aircraft is approximated as a point mass, and its positions in two-space is given by parametric equations describing the movement of a point.

The procedure is to first consider the points moving on the infinite straight lines determined by the segments. The time the two points are at a minimum distance can be determined by the derivative. Check to see if this time lies between the begin-time and end-time for the interval under consideration. Last, check the distance at the end-points: the start-time and the end-time.

The technical details for taking the derivative are: Given the start and end points of a vector, get the sine and cosine of the vector. Suppose $\left(\mathrm{x}_{\mathrm{i}}, \mathrm{y}_{\mathrm{i}}\right)$ is the start point for vector $\mathrm{i}$. The parametric equations for a point traversing the line determined by vector $i$ with angle $\theta_{i}$ at velocity $v$ are

$$
\begin{aligned}
& \mathrm{x}_{\mathrm{i}}+\mathrm{tr} \cos \theta_{\mathrm{i}} \\
& \mathrm{y}_{\mathrm{i}}+\mathrm{tr} \sin \theta_{\mathrm{i}}
\end{aligned}
$$

The square of the distance between two points at time $t$ is

$$
\begin{aligned}
\mathrm{s}^{2}= & {\left[\mathrm{x}_{1}+\mathrm{tv} \cos \theta_{1}-\mathrm{x}_{2}-\mathrm{tv} \cos \theta_{2}\right]^{2} } \\
& +\left[\mathrm{y}_{1}+\mathrm{tr} \sin \theta_{1}-\mathrm{y}_{2}-\mathrm{tr} \sin \theta_{2}\right]^{2}
\end{aligned}
$$

Taking the derivative of the square of the distance with respect to $t$, setting the derivative equal to zero, and solving for $t$ gives

$$
\begin{aligned}
& \mathrm{t}=\mathrm{num} / \mathrm{den} \\
& \mathrm{num}=-\left(\mathrm{x}_{1}-\mathrm{x}_{2}\right)\left(\cos \theta_{1}-\cos \theta_{2}\right) \\
&-\left(\mathrm{y}_{1}-\mathrm{y}_{2}\right)\left(\sin \theta_{1}-\sin \theta_{2}\right) \\
& \text { den }= \mathrm{v}\left(\cos \theta_{1}-\cos \theta_{2}\right)^{2} \\
&+\mathrm{v}\left(\sin \theta_{1}-\sin \theta_{2}\right)^{2}
\end{aligned}
$$

The second derivative is negative. Hence, this gives a minimum for the infinite lines through the segments.

\section{DESCRIPTION AND CONSTRUCTION OF A JOG}

This section describes a jog to the left. The jog begins a distance of two units from the point of minimum distance. First, a turn of $\pi / 4$ radians is made to the left. This flight segment is $2 \sqrt{2}$ units in length. The program computes the begin and end time and points for this flight segment. Second, a turn of $\pi / 2$ radians is made to the right. This flight segment is $2 \sqrt{2}$ units in length. The program computes the begin and end time and points for this flight segment. Third, a turn of $\pi / 4$ radians is made to the left. This places the aircraft back on its original path. The aircraft will have the same exit point, but exit will be delayed because of the longer flight path. The program computes the begin and end time and points for this flight segment.

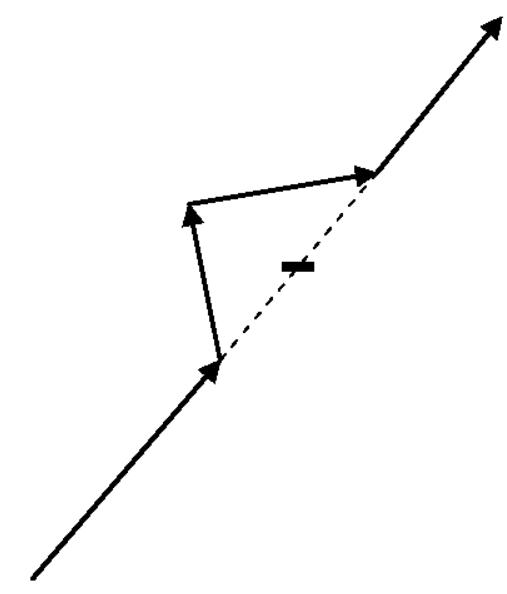

Figure 2 - The four segments of a jog to the left.

The primary computational tools for the jog are the sine and cosine. Suppose $\theta$ is the angle of the original flight path. 
For the first segment of the jog:

$$
\begin{aligned}
& \sin (\theta+\pi / 4)=(\sin \theta+\cos \theta) / \sqrt{2} \\
& \cos (\theta+\pi / 4)=(\cos \theta-\sin \theta) / \sqrt{2}
\end{aligned}
$$

For the second part of the jog:

$$
\begin{aligned}
& \sin (\theta-\pi / 4)=(\sin \theta-\cos \theta) / \sqrt{2} \\
& \cos (\theta-\pi 4)=(\cos \theta+\sin \theta) / \sqrt{2}
\end{aligned}
$$

The start-time and start-point of the first segment of the jog are known. The sine and cosine along with the velocity are used to compute the end-time and end-point. These become the start-time and start-point of the second segment. Similarly for the final segment that lies on the original flight path.

To check that collision-avoidance has been achieved, the four segments of the new flight path are each compared to the flight path of the other aircraft to check for closeness. In general, each of the four segments of the aircraft making the jog will have a start-time and end-time that is different from the other aircraft, but the routine for checking closeness is general enough to handle this.

\section{Types of Collision Avoidance}

There are five collision avoidance routines.

If the angle between the flight paths is less than $\pi / 2$ radians, then the second aircraft dithers for $4 / 96$ of an hour. This places it 4 units (20 nautical miles) later on its original flight path. The justification for this maneuver is the twodimensional nature of the simulation. The two flight paths could be nearly coincident, neither aircraft can fly over or under the other, and the only option is to delay the flight of one of the aircraft.

There are four choices if the angle between the flight paths is greater than or equal to $\pi / 2$ radians. The exposition below describes the two options where the second aircraft is approaching the first and the second aircraft is originally to the left of the first.

The first option occurs when the flight path of the second aircraft intersects the path of the first aircraft after the point where the two aircraft are a minimum distance apart. In this case, the second jogs to its right. This is illustrated in figures three through six.

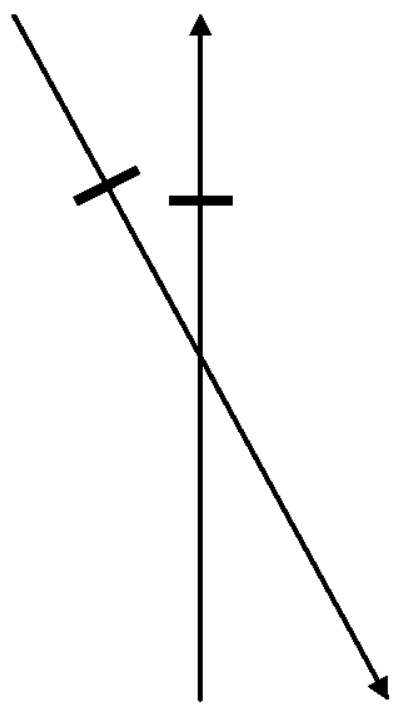

Figure 3 - Flight path of second aircraft intersects path of first aircraft after point of min distance

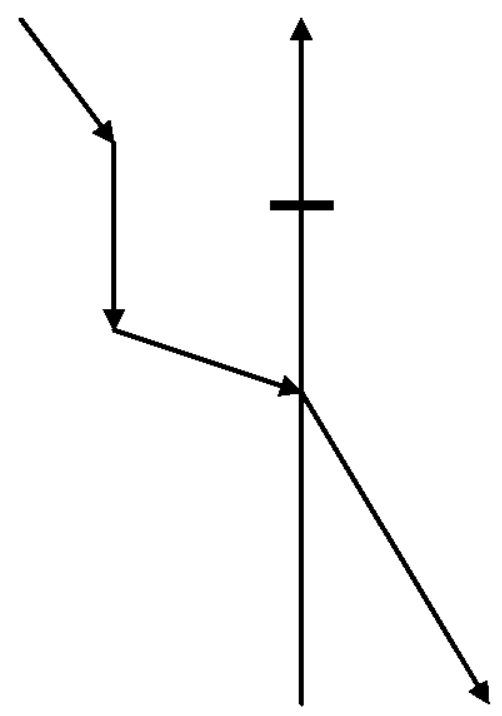

Figure 4 - If flight path of second aircraft intersects path of first aircraft after point of min distance, second aircraft jogs to its right

The second option occurs when the flight path of the second aircraft intersects the path of the first aircraft before the point where the two aircraft are a minimum distance apart. In this case, the second jogs to its left. This is illustrated in figures 


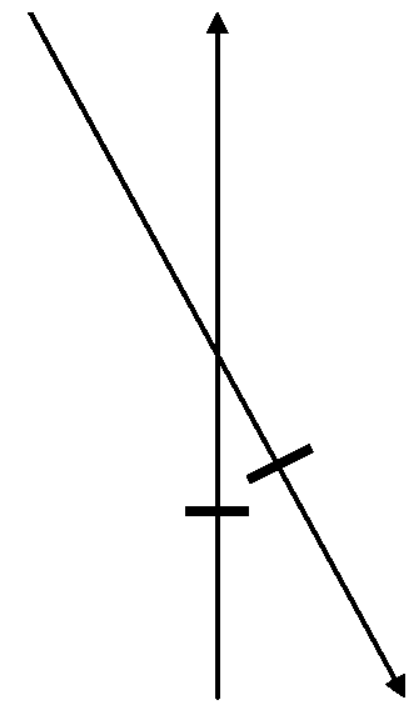

Figure 5 -Flight path of second aircraft intersects path of first aircraft before point of min distance

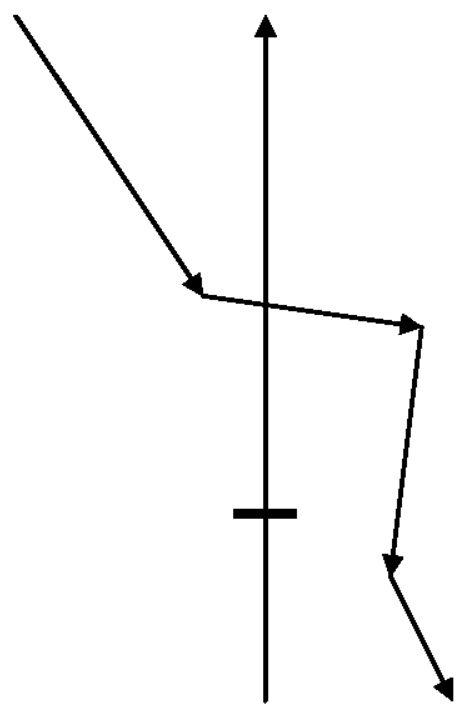

Figure 6 -If flight path of second aircraft intersects path of first aircraft before point of min distance, second aircraft jogs to its left

A conjecture which has not been tested is that the above rules can be simplified. In the cases above the aircraft performing the maneuver jogs away from the other aircraft. Can the rule be simplified to determining whether the other aircraft is on the left or right at the point of minimum distance?

\section{DesCRiption of Simulation SPaCE}

The airspace in this simulation was chosen to be an idealized airspace, based on the statistic that most aircraft spend about 20 minutes in any one airspace during a flight.

The requirement for horizontal separation is 5 nautical miles. Hence, each unit distance represents 5 nautical miles.

Aircraft in cruise mode typically maintain a constant speed. The airspeed chosen is 96 units per hour (580 knots).

The airspace is a circle of radius 16 . Hence, an aircraft spends 20 minutes traversing its diameter.

The initial flight path for an aircraft is a straight line. Collision avoidance consists of either a delay before entering the airspace or adding two line segments for a jog and returning to the original path. The extra distance due to collision avoidance does delay the exit of an aircraft from the airspace.

Each trial consists of simulating two aircraft with the initial flight path of the second chosen to come within distance 1 of the first aircraft.

The first aircraft traverses the diameter of the circle beginning at the bottom of the circle.

The path of the second aircraft is determined by choosing two points at random. The first point is on the circumference of the airspace. The second point is within a distance of 1 from the first plane at some time during its sojourn.

For the first point, an assumption is that previous airspace operations have kept the aircrafts well separated. Therefore, the start point for the second aircraft is a point on the circle that is greater than two units (measured along the circumference) from the start point of the first aircraft. This point is chosen using the uniform distribution.

The second point is chosen using three random numbers from the uniform distribution.

The first aircraft traverses the airspace across the circle from $(0,-16)$ to $(0,+16)$. The first random number is chosen uniformly on the line segment $(0,-14)$ to $(0,+14)$. This will be the position of the first aircraft when the second aircraft would have reached the second point to be chosen if the second aircraft had not modified it flight path. 
The second point is chosen within a circle of radius 1 with the center being the randomly chosen position of the first aircraft. It is a two step procedure. First, chose uniformly from the angles from 0 to 2 pi radians. Second, chose uniformly from the numbers 0 to 1 .

The second point chosen is not necessarily the point of minimum distance for the two aircrafts. In fact, it will usually not be.

Figure 1 illustrates the flight path of the first aircraft and choosing the two points that determine the path of the second.

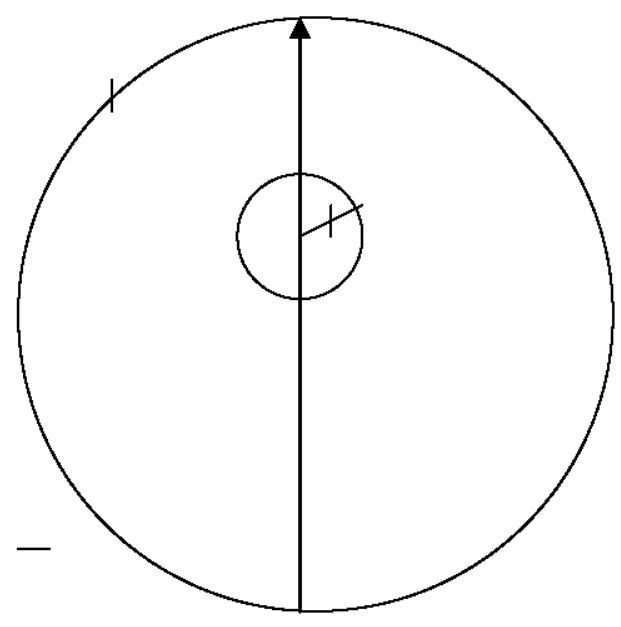

Figure 7 - The flight path of the first aircraft and choosing the two points that determine the path of the second aircraft

Figure 2 illustrates the original, planned flight path of the two aircraft. The second plane will make a collisionavoidance jog.

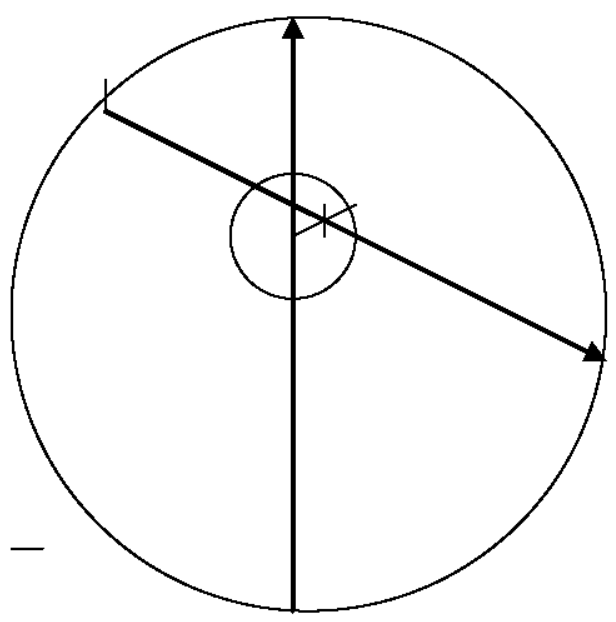

Figure 8 - The flight path of the two aircrafts

The entry time of the second aircraft is chosen so that it arrives at the second point within the smaller circle at the same time the first aircraft arrives at the center of the circle (which lies on its flight path).

It can be seen that the second point within the smaller circle may not be the point at which the aircrafts are a minimum distance apart.

It can be seen that the initial flight path of the second aircraft can be longer than the flight path of the second aircraft. In this case, the second aircraft enters first, even though it is the one that takes evasive maneuvers.

\section{Simulation Runs}

The procedures above were implemented as a set of MATLAB m-files. These programs were extensively tested to ascertain they generated the intended flight paths, included the required jogs, and detected any loss of separation. A full report would include the test cases. It takes six hours to run one hundred million $(100,000,000)$ trials.

The program is efficient enough to generate enough trials for statistical significance. More than a billion trials have been made, and the algorithm has performed correctly for every trial. This is efficient enough to establish statistically significant results, as will be seen in the next two sections. 


\section{Elementary Probability Analysis}

The FAA goal is one incident per 10 million $\left(10^{7}\right)$ flights. As it stands, this goal is not a probability statement, and it requires interpretation. In the absence of information and for simplicity, the typical assumption is that all flights are equivalent and independent. The typical numerical assumption is that the expected number of incidents for 10 million flights be equal to one. Using the expectation does not require any more information from the FAA, but it does have a disadvantage as will be seen below.

The disadvantage appears when we consider the probability of more than one incident during 10 million flights. It's reasonable to want the probability of more than one incident to be low, but it will be shown that using the expectationinterpretation does not guarantee this. On the other hand, the low-probability approach raises the problem of how low the FAA wishes the probability to be.

With the assumption that the flights are equivalent and independent, the distribution is binomial with the probability of an incident equal to $10^{-7}$ per flight. The binomial distribution with parameter $\mathrm{p}$ gives the probability of zero or one incidents during 10 million flights as

$\mathrm{Q}=(1-\mathrm{p})^{10000000}+10000000 \mathrm{p}(1-\mathrm{p})^{9999999}=0.7358$

The probability of two or more incidents for $\mathrm{p}=1 \mathrm{e}-7$ is $1-\mathrm{Q}$ $=0.2642$. Hence, the probability of more than one incident during 10 million flights is greater than $1 / 4$.

If the goal is a less than one in a hundred chance of more than one incident, then a little numerical work gives $\mathrm{Q}=$ 0.9898 and $1-\mathrm{Q}=0.0102$ for $\mathrm{p}=1.5 \mathrm{e}-8$.

We wish to determine the number of trials needed for an experiment to establish the probability of failure is less than or equal to $\mathrm{p}$ at the $100(1-\alpha) \%$ confidence level given no failures appears during the experiment.

The frequency interpretation of the confidence level is that the experiment has an $\alpha$ or less chance of misleading us. Hence, we conduct enough trials $\mathrm{n}$ that the probability that no failures appear is less than $\alpha$ given the probability of failure is less than or equal to $\mathrm{p}$. That is,

$$
(1-p)^{\mathrm{n}} \leq \alpha
$$

or

$$
\mathrm{n} \geq(\log \alpha) /(\log (1-\mathrm{p}))
$$

We will ask for a confidence level commensurate with the probability we are seeking to establish and set $\alpha=p$. We solve for $\mathrm{n}$ setting the last inequality to an equality.

If $\alpha=\mathrm{p}=1 \mathrm{e}-7, \mathrm{n}=1.6118 \mathrm{e}+8=161,180,000$
If $\alpha=\mathrm{p}=1.5 \mathrm{e}-8, \mathrm{n}=1.2010 \mathrm{e}+9=1,201,000,000$

Since 100 million trials requires six hours of computer time, the first experiment required one overnight run, and the second experiment required six overnight runs. All runs were successful. Hence, the algorithm has been statistically verified according to two reasonable interpretations of the FAA requirement

\section{Field Data and Conditional Probability}

This section offers a more realistic analysis. First, there are causes for incidents other than loss of separation. Flight data has not been examined, but hypothetically assume one out of every hundred incidents is due to loss of separation. This makes verification more stringent. Using elementary results on adding probabilities and combining confidence levels says we now need to establish the algorithm at a 1e-9 probability with a confidence level of $100(1-1 \mathrm{e}-9) \%$ [2].

Not every fight, however, has the potential for a collision incident. Table 1 displays the pilot reported near mid-air collisions for the last seven years [8]. The average is 168.6.

\section{Table 1: Pilot Reported} Near Mid-Air Collisions

\begin{tabular}{|c|c|}
\hline Year & Reported Number \\
\hline 2000 & 239 \\
\hline 2001 & 211 \\
\hline 2002 & 180 \\
\hline 2003 & 162 \\
\hline 2004 & 145 \\
\hline 2005 & 138 \\
\hline 2006 & 105 \\
\hline
\end{tabular}

The conditional probability statement is

$\mathrm{P}($ collision $)$

$=\mathrm{P}($ collision given loss of separation $)$

$\mathrm{x} \mathrm{P}($ loss of separation $)$

Recall we want $\mathrm{P}$ (collision) $=1 \mathrm{e}-9$.

To establish $\mathrm{P}$ (collision) is less than 1e-9 at the $100(1-1 \mathrm{e}-$ $9) \%$ confidence level, it is sufficient that the two factors give a multiple less than $1 \mathrm{e}-9$ and the two factors have a $100(1-5 \mathrm{e}-10) \%$ confidence level.

This approach uses the elementary result on multiple confidence levels that if the statistics have a confidence level of $100\left(1-\mathrm{h}_{1}\right) \%$ and $100\left(1-\mathrm{h}_{2}\right) \%$, then they have a joint confidence level of $100\left(1-h_{1}-h_{2}\right) \%$. 
The usual method of establishing a confidence interval is by the Central Limit Theorem, but this is a convergence approximation, and it's not certain it can be trusted at the extremely high level of $100(1-5 \mathrm{e}-10) \%$. Hence we will proceed with approximate, conservative figures and conservative methods.

The distribution is binomial. There are more than $1 \mathrm{e}+7$ flights per year. We ask what binomial parameter $\mathrm{p}$ gives less than a $5 \mathrm{e}-10$ chance of $200(>168)$ or less events in $1 \mathrm{e}+7$ trials.

Since the terms in the binomial expansion are monotonically increasing for the first pe +7 terms [1], we have

$$
\begin{aligned}
& \sum_{k=0}^{200}\left(\begin{array}{c}
1 e+7 \\
k
\end{array}\right) p^{k}(1-p)^{1 e+7-k} \\
& \leq 201\left(\begin{array}{c}
1 e+7 \\
200
\end{array}\right) p^{200}(1-p)^{1 e+7-200} \\
& =201\left\{\frac{1 e+7}{200} p(1-p)^{49999}\right. \\
& \left.\times \cdots \times \frac{1 e+7-199}{1} p(1-p)^{49999}\right\}
\end{aligned}
$$

The expansion is for computational purposes since $1 \mathrm{e}+7$ factorial is too large for straightforward calculation.

For $\mathrm{p}=1 \mathrm{e}-4=\mathrm{P}$ ( loss of separation), the expression above is less than $1.25 \mathrm{e}-207$ which is less than $5 \mathrm{e}-10$. Solving equation (8) above gives $\mathrm{P}$ (collision given loss of separation $)=1 \mathrm{e}-5$. Establishing a probability of $1 \mathrm{e}-5$ at the $100(1-5 \mathrm{e}-10) \%$ confidence level requires $\mathrm{n}=(\log (5 \mathrm{e}-$ $10)) /(\log (1-1 \mathrm{e}-4))=2.14 \mathrm{e}+6$ trials.

The last computation was conservative because it used a confidence level based on $5 \mathrm{e}-10$. It could have used a confidence interval based on ( $1 \mathrm{e}-9-1.25 \mathrm{e}-207)$.

We might ask the number of trials required if one out of every thousand incidents was due to loss-of-separation or if the required probability of an incident was $1.5 \mathrm{e}-8$ as derived in the previous section. Table 2 summarizes the results with the second column repeating the computation above. Table 2 continues the conservative method of determining the confidence level.
Table 2: Number of Trials Required Given Various Probability Requirements

\begin{tabular}{|l|l|l|l|l|}
\hline $\begin{array}{l}\text { Desired } \\
\text { Probability } \\
\text { of Incident } \\
\text { Per Flight }\end{array}$ & $1 \mathrm{e}-7$ & $1 \mathrm{e}-7$ & $1.5 \mathrm{e}-8$ & $1.5 \mathrm{e}-8$ \\
\hline $\begin{array}{l}\text { Fraction of } \\
\text { Incidents } \\
\text { Allotted to } \\
\text { Collision }\end{array}$ & 0.010 & 0.001 & 0.010 & 0.001 \\
\hline $\begin{array}{l}\text { Required } \\
\text { Conditional } \\
\text { Probability } \\
\text { of Collision }\end{array}$ & $1 \mathrm{e}-5$ & $1 \mathrm{e}-6$ & $1.5 \mathrm{e}-6$ & $1.5 \mathrm{e}-8$ \\
\hline $\begin{array}{l}\text { Required } \\
\text { Confidence } \\
\text { of Cond } \\
\text { Prob }\end{array}$ & $5 \mathrm{e}-10$ & $5 \mathrm{e}-11$ & $7.5 \mathrm{e}-11$ & $7.5 \mathrm{e}-12$ \\
\hline $\begin{array}{l}\text { Number of } \\
\text { Trials } \\
\text { Required } \\
\text { To }\end{array}$ & $2.1 \mathrm{e}+6$ & $2.4 \mathrm{e}+7$ & $2.3 \mathrm{e}+7$ & $2.6 \mathrm{e}+8$ \\
$\begin{array}{l}\text { Establish } \\
\text { Cond Prob } \\
\text { at Conf } \\
\text { Level }\end{array}$ & & & & \\
\hline
\end{tabular}

It can be seen that conditioning both increases the realism and reduces the experimental effort. This is a welcome result since more realistic simulations are likely to be less efficient than this first effort.

\section{FURTHER WORK}

The narrative above has identified several places where even this simple program could be made more efficient. The algorithm can be tested when more than two aircraft are in the airspace. Are there situations or is there a number of aircraft for which this algorithm no longer works. Actual traffic can be observed or simulated to get the distribution of flight paths for loss-of-separation incidents. Is it possible to conduct exhaustive testing? Perhaps a variation of less than one degree in a flight path does not affect the algorithm. A three dimensional algorithm can be designed and tested. The avoidance jogs can be made more realistic. The study can be more global by considering the problem for adjoining airspaces. A major element not included in this work is uncertainty and perturbations. These items may represent the greatest challenge to being both realistic and efficient. Finally, the current statistical methods are effective but could use refinement. 


\section{SUMMARY}

This paper describes a first effort at designing and testing a collision avoidance algorithm. Designing the algorithm was an exercise in geometry. Implementing in a program for automatic testing was an exercise in analytic geometry, trigonometry, and calculus.

One goal was to try the popular and portable platform of MATLAB for the simulation. To date, this has worked well. The program was efficient enough to perform many trials in a short period of time. The current question is whether or not this efficiency can be maintained as the simulation is made more realistic.

This effort was initially performed without reference to any other work to force the author to think through everything although the effort was motivated by the author's impression of what work had been done. In particular, there was no mention of a collision-avoidance algorithm being established at an extremely high confidence level $[3,4,5]$. Because of the enormous number of trials involved, there are efforts to rigorously prove the algorithms [6], but it is unclear if the proofs can be extended to include perturbations.

\section{REFERENCES}

[1] William Feller, "An Introduction to Probability Theory and It's Applications", Volume I, Wiley, New York, 1968.

[2] Samuel Wilks, "Mathematical Statistics", Wiley, New York, 1963.

[3] J. Kucher and L. Yang, "Survey of conflict detection and resolution modeling methods," AIAA Guidance, Navigation, and Control Conference, Volume AIAA97-3732, pp. 1388-1397, New Orleans, 1997.

[4] K. Bilimoria, "A geometric optimization approach to aircraft conflict resolution," Guidance, Navigation, and Control Conference, Volume AIAA-2000-4265, Denver, 2000.

[5] B. Abdul-Baki, J. Baldwin, and M-P. Rudel, "Independent validation and verification of the TCAS II collision avoidance subsystem," IEEE AES Systems Magazine, August 2000.

[6] J. Lygeros and N. Lynch, "On the formal verification of the TCAS conflict resolution algorithms," Proceedings $36^{\text {th }}$ IEEE Conference on Decision and Control, San Diego, pp. 1829-1834, December 1997. [8]http://www.bts.gov/publications/national_transportation statistics/html/table_02_15.html

\section{BIOGRAPHY}

The author is a research mathematician at NASA Langley research centers. His previous work and publications include probability modeling, design of experiments, stochastic control, and testing software. 